\title{
Infection control in acute care facilities: Evidence-based patient safety
}

\author{
Lindsay E Nicolle MD FRCPC, Editor-in-Chief, University of Manitoba, Winnipeg, Manitoba
}

$\mathrm{I}^{\mathrm{n}}$ nfection control in acute care facilities has a noble history. 1 These programs were born of the nosocomial penicillinresistant Staphylococcus aureus outbreaks in the post-World War II era. Over the past four decades, an impressive body of evidence has emerged that documents the effectiveness of infection control programs and systematically evaluates specific program components. Fumigation, tacky floor mats, shoe covers and 'reverse' isolation have disappeared. They are replaced by focused surveillance programs, prophylactic antimicrobial therapy, outbreak investigation and control, routine barrier practices and molecular typing of organisms for epidemiological analysis.

The first quarter-century of infection control culminated in the Study on the Efficacy of Nocomial Infection Control, which unequivocally answered the question: 'What is the value of an infection control program?' (1). In the early 1980s, an effective infection control program - defined by an appropriately trained clinician leader, one infection control practitioner for 250 beds and a nosocomial surveillance program including surgical wound infection rate feedback to surgeons - decreased the prevalence of endemic nosocomial infections in acute care facilities by $30 \%$ to $50 \%$. Evolution in health care practice continues to necessitate evaluation of specific control interventions, but the effectiveness of an infection control program in an acute care facility is clear. Prevention of nosocomial infections decreases mortality, lengths of stay, the need for antimicrobial therapy, and surgical and other invasive interventions. In the current fiscal environment, infection control programs have repeatedly been shown to be cost effective $(2,3)$.

In an era of evidence-based medicine and cost effectiveness, why does infection control so frequently seem to be under siege when it is supported by both convincing evidence and clear cost effectiveness? Canadian infection control programs are frequently under-resourced. There are inadequate numbers of trained personnel and limitations in essential supports, including computer access and clinical laboratory support. Infection control has repeatedly been vulnerable during health care restructuring. Activities and personnel are combined with other programs, or positions are simply eliminated. Where regionalization and integration of service delivery have progressed, the additional infection control activity for the development and operation of regional programs with cross-facility standardization and benchmarking has not been acknowledged or supported. On the other hand, when the value of infection control programs has been acknowledged, proposals to incorporate infection control with other programs with different knowledge, skill and evidence bases have undermined infection control effectiveness by diffusing resources. For instance, it has been proposed that antimicrobial resistance containment should be an infection control function. There is increasing attention to what are called 'medical errors', with nosocomial infections being included in the list of such errors, and suggestions to merge infection control into comprehensive programs to prevent medical errors. This is reminiscent of proposals to merge infection control with 'quality assurance' in the past decade. Finally, the proven effectiveness of infection control in acute care facilities has resulted in regionalized health services expanding infection control activity to long term care or community services, without providing additional resources to support or validate this activity.

of course, difficulty in achieving support for infection control is the Catch-22 inherent in all prevention activity. If effective, the outcome is negative, ie, no infection. Thus, the program is not seen to provide a service and is undervalued. Infection control in acute care facilities must be acknowledged for what it is - an evidence-based, cost effective prevention activity that promotes patient and staff safety. There is a unique body of knowledge - clinical, microbiological, epidemiological and patient care - as well as leadership and communication skills, which are essential for effective infection control. As health care delivery evolves, this uniqueness 
of infection control must be acknowledged, and the integrity of these programs in acute care facilities must be maintained and supported.

We also need to acknowledge the boundaries of infection control. Infection control is not an antimicrobial resistance containment program. While infection control activity is one important element of antimicrobial resistance control, this role should be minor. Antimicrobial use is the driving determinant of antimicrobial resistance, and antimicrobial use programs are necessary to optimize practice. This must remain the responsibility of the medical staff. The role of infection control is to limit the transmission of resistant organisms once they have emerged at a facility and is not effective in the absence of an effective antimicrobial use program. Nosocomial infections may or may not be considered under the umbrella of 'medical errors'; some infections are not preventable despite optimal practice. Certain elements of infection control practice, including an expert knowledge base, use of epidemiological principles and practice for surveillance and containment, and cost effective analysis, are applicable generally to medical errors. However, infection control also addresses the unique problems of transmissible organisms, with a possible negative impact not just for the single patient in whom a 'mistake' is made, but for other patients and staff as well. Finally, the practice of infection control in acute care facilities has been documented to be effective, but may not be relevant to other health care delivery sites. Barrier precautions appropriate for the care of patients in intensive care units are not appropriate in long term care facilities, and to date, there is no evidence that improved handwashing limits infections in out-of-hospital settings. Infection control interventions have yet to be validated in health care settings outside acute care.

What should be included in an infection control program in an acute care facility (4)? First, it is key to have an active system for surveillance of nosocomial infections that is relevant to the institution, valid and reviewed regularly, and in which data are interpreted and communicated in a timely fashion. Potential outbreaks must be recognized early, and appropriate investigation and control activity must be implemented promptly. Infection control programs should provide expert health support for staff in matters relevant to infections in staff members, or transmission of infections between patients and staff. Appropriate policies and procedures for patient care practices to minimize the acquisition of nosocomial infections must be developed, updated regularly and monitored for compliance. These include handwashing, outbreak control, isolation practices, use of invasive devices, and environmental issues related to housekeeping, linen management, food preparation, and disinfection and sterilization of equipment. Infection control staff must liaise effectively with public health, and communicate with other facilities and individuals, providing health services where appropriate. Finally, infection control should participate in programs for the education of patients, staff, family and others on issues relevant to the control of nosocomial infections.

Infection control must have appropriate resources to perform these activities. These resources include effective, active leadership, as well as appropriate numbers of personnel, an administrative reporting structure that supports the program, adequate clinical laboratory support for identifying and reporting isolates from clinical specimens, as well as typing for epidemiological investigations, and appropriate office resources, including computer support.

In exchange for this, patients, clinicians, facilities and funders of acute care facilities can demand certain 'deliverables' from infection control. They should expect, overall, an infection control program that maximizes patient and staff safety, and is cost efficient. They have a right to documentation of the effectiveness of the program through, for example, surveillance of endemic infections, reports of outbreak investigation and control, evaluations of new equipment and new procedures, and updated and timely policies and procedures. They have a right to information documenting compliance with practice. Finally, they should expect the infection control team and individual members to participate in a collaborative fashion with other groups and individuals, both inside and outside the facility, to promote health service delivery.

These are stressful times for health care in Canada. Some of the stressors, such as limited health care personnel and resources for infrastructure, as well as future uncertainty, negativly impact patient care. This is seen in prolonged waiting lists, overcrowded emergency wards, readmission of patients following early discharge and resources insufficient for support services such as rehabilitation or even basic ward equipment. For hospital infections, it is seen in changing staffing ratios, decreased frequency of environmental cleaning and underfunding of infection control programs. As we work through structures and restructures to maintain an effective health care system for Canada, it is important to remain pragmatic and remember to 'go where the money is'. For patient safety in acute care facilities, the money is clearly in infection control. We need to acknowledge this, and ensure infection control programs are appropriately recognized and supported to achieve common goals in quality patient care.

\section{REFERENCES}

1. Haley RW, Culver DH, White JW, et al. The efficacy of infection surveillance and control programs in preventing nosocomial infections in US hospitals. Am J Epidemiol 1985;121:182-205.

2. Wenzel RP. The economics of nosocomial infections. J Hosp Infect 1995;31:79-87.

3. Haley RW, White JW, Culver D, Hughes JM. The financial incentive for hospitals to prevent nosocomial infections under the prospective payment system. J Am Med Assoc 1987;257:1611-4.

4. Scheckler WE, Brimhall D, Buck AS, et al. Requirements for infrastructure and essential activities of infection control and epidemiology in hospitals: a consensus panel report. Society of Healthcare Epidemiology of America. Infect Control Hosp Epidemiol 1998;19:114-24. 


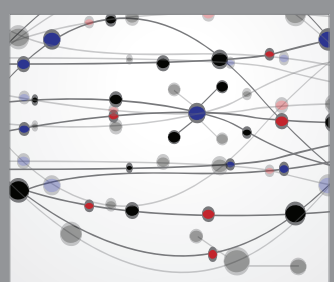

The Scientific World Journal
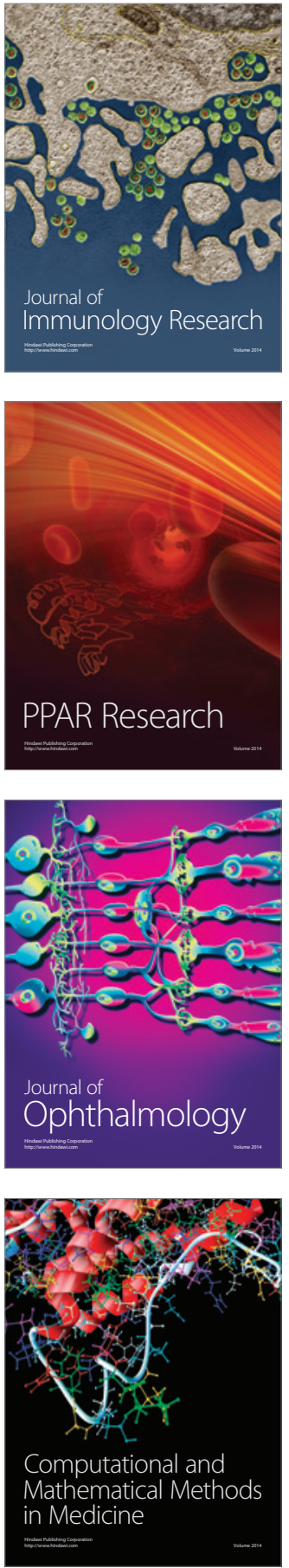

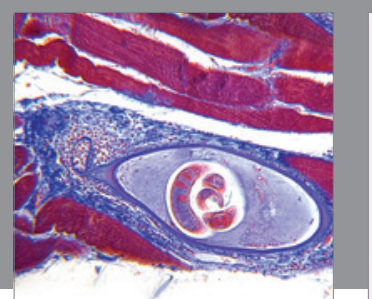

Gastroenterology Research and Practice

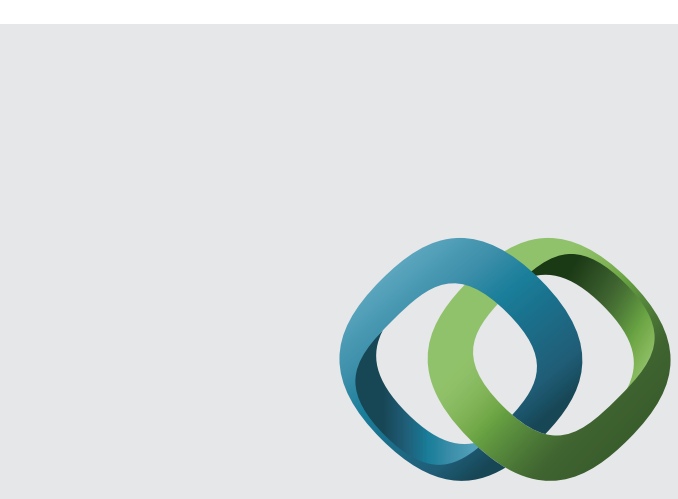

\section{Hindawi}

Submit your manuscripts at

http://www.hindawi.com
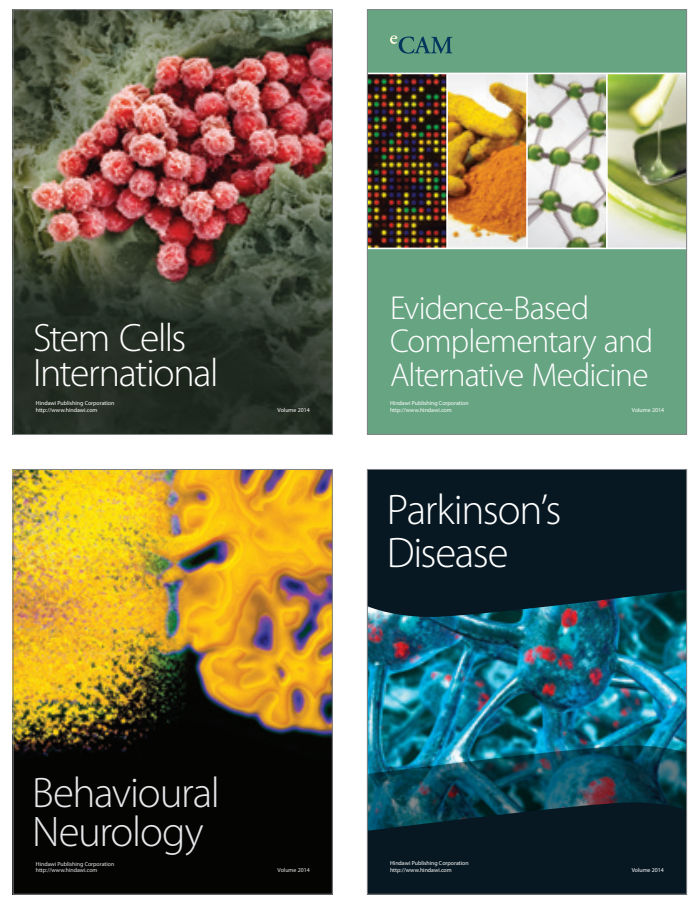
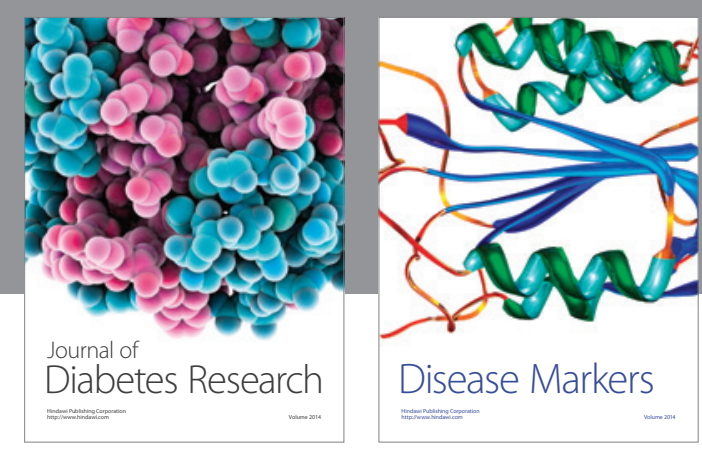

Disease Markers
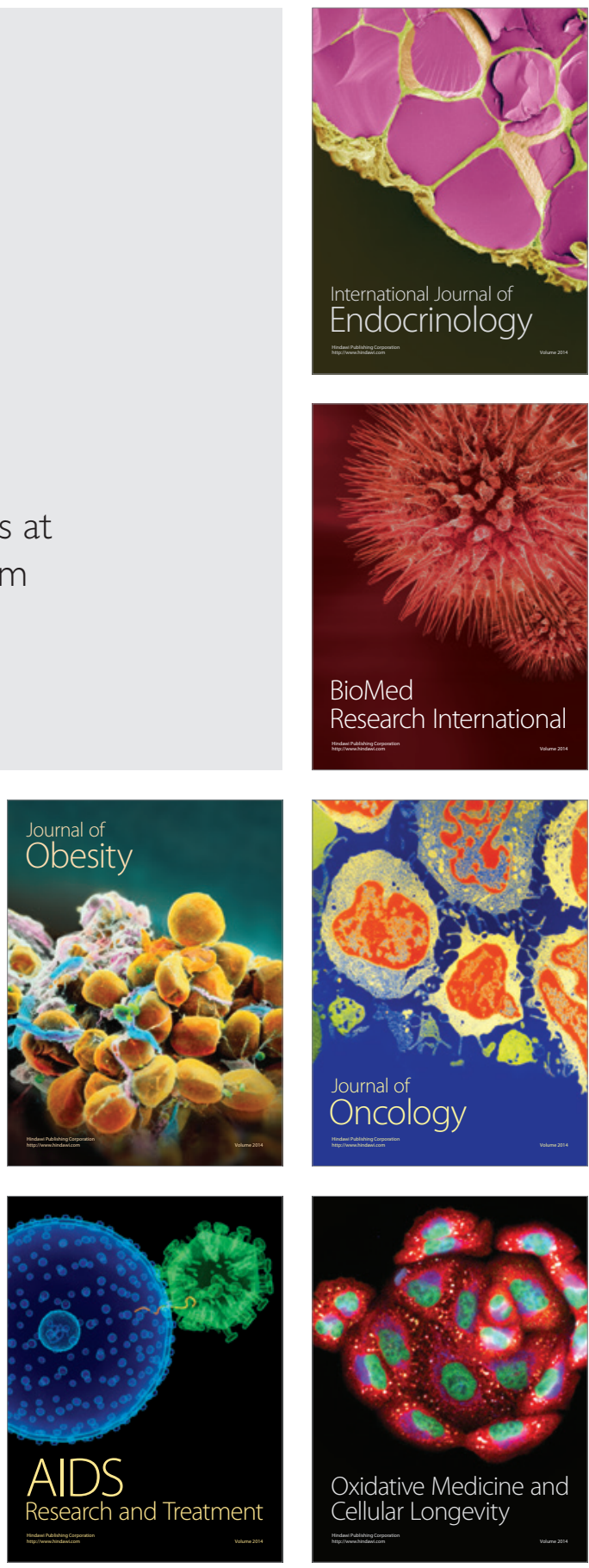